\title{
Erratum to: Recommendations for the clinical and radiological evaluation of response to treatment in metastatic renal cell cancer
}

Luís León • Roberto García-Figueiras • Cristina Suárez • Antonia Arjonilla • Javier Puente • Blanca Vargas •

Maria José Méndez Vidal • Carmen Sebastiá

Published online: 3 May 2014

(C) Springer International Publishing Switzerland 2014

Erratum to: Targ Oncol (2014) 9:9-24

DOI 10.1007/s11523-013-0304-7

An error was subsequently identified in the article, and the following correction should be noted:

The name of the 2nd author, which previously read: "Roberto García-Figueras" should read: "Roberto García-Figueiras"

The online version of the original article can be found at http://dx.doi.org/ 10.1007/s11523-013-0304-7.

\section{León}

Medical Oncology Department, Complejo Hospitalario Universitario

de Santiago, A Coruña, Spain

R. García-Figueiras

Radiology Department, Complejo Hospitalario Universitario de

Santiago, A Coruña, Spain

C. Suárez

Medical Oncology Department, Hospital Universitari Vall

D'Hebron, Barcelona, Spain

A. Arjonilla

Radiology Department, Hospital Universitario Rey Juan Carlos,

Madrid, Spain

J. Puente

Medical Oncology Department, Hospital Clínico San Carlos,

Madrid, Spain
B. Vargas

Radiology Department, Hospital Universitario Virgen del Rocío,

Sevilla, Spain

M. J. Méndez Vidal

Medical Oncology Department, Hospital Universitario Reina Sofia,

Córdoba, Spain

C. Sebastiá

Radiology Department, Hospital Universitari Clinic, Barcelona, Spain

L. León $(\bowtie)$

Medical Oncology Department, Hospital Clínico Universitario de Santiago, C/ Choupana S/N, Santiago de Compostela 15706, Spain e-mail: luis.leon.mateos@sergas.es 